\title{
Quantum tunneling of magnetization in single domain particles
}

J. Tejada and LI. Balcells

Facultat de Fisica, Dept de Física Fonamental, Universitat de Barcelona, Diagonal,

647, 08028 Barcelona, Spain

S. Linderoth

Laboratory of Applied Physics, Technical University, Denmark DK*2800 Lyugby

R. Perzynski

Université Pierre and Marie Curie (Paris 6), 75252 Paris, Cedex 05, France

B. Rigau

Facultat de Física, Dept. de Fisica Fonamental, Universitat de Barcelona, Diagonal, 647, 08028 Barcelona, Spain

\section{B. Barbara}

Laboratoire de Magnetism Louis Néel, 38100 Grenoble, Cedex, France

J. C. Bacri

Université Pierre and Marie Curie (Paris 6), 75252 Paris, Cedex 05, France

We present a study of the magnetic relaxation of several ferrofluids composed of particles of about $40 \AA$ in diameter $\left(\mathrm{Fe}_{3} \mathrm{O}_{4} \mathrm{FeC}, \mathrm{CoFe}_{2} \mathrm{O}_{4}\right)$. Our key observation is a nonthermal character of the relaxation below $3 \mathrm{~K}$ for the $\mathrm{CoFe}_{2} \mathrm{O}_{4}$ ferrofluid and below $1 \mathrm{~K}$ for the $\mathrm{FeC}$ ferrofluid. The crossover temperature from thermal to nonthermal (quantum) regime is in accordance with theoretical suggestions of macroscopic quantum tunneling of magnetization in single domain particles.

Are mesoscopic degrees of freedom governed by quantum mechanics? According to Leggel ${ }^{1}$ we discuss in this paper the dynamics of the magnetization $M$ of small single domain particles as governed by quantum mechanics below a certain critical temperature. ${ }^{2}$

Let us assume that the magnetic moment of one of these single domain particles looks along the $Z$ axis and the field is applied in the opposite direction. The stable magnetic state corresponds to $M$ directed along $H$. To rotate an individual atomic magnetic moment with respect to others costs the exchange energy $\epsilon_{\mathrm{ex}}$ per atom. To coherently rotate the total magnetic moment of the particle out of the anisotropy axis costs $\epsilon_{\mathrm{an}} N$, where $\epsilon_{\mathrm{an}}$ is the anisotropy energy and $N$ is the total number of magnetic atoms. Thus, at $N<\epsilon_{\mathrm{ex}} \cdot \epsilon_{\mathrm{an}} \sim 10^{3}-10^{5}$ it is energetically favorable to develop a uniform rotation of $M$ towards the minimal energy state.

The energy barrier, $U$, for coherent magnetization rotation of a single domain particle is determined by the magnetic anisotropy and the external applied field ${ }^{3,4}$

$$
U=k V\left(1-H / H_{0}\right)^{2},
$$

where $k$ is the anisotropy constant, $V$ and $M$ are the volume and the magnetization of the switching unit, and $H$ is the applied field and $H_{0}=2 k / M$. The switching rate, $\Gamma$, for thermal activated processes decreases exponentially with the ratio of the energy barrier $U$ and the thermal energy $k_{B} T$

$$
\Gamma_{T}=v \exp \left(-U / k_{B} T\right),
$$

where $v$ is usually of the order of the ferromagnetic resonance frequency, $v \sim 10^{10}-10^{11} \mathrm{~s}^{-1}$. The thermal activated processes vanish in the limit of zero absolute temperature.
It has been theoretically predicted ${ }^{2}$ that below a characteristic (crossover) temperature, $T_{c}$, the escape of $M(r)$ from metastable configurations is dominated by quantum underbarrier transitions, not by thermal overbarrier activation. In the usual Wentzel-Kramers-Brillouin approximation, the transition rate $\Gamma_{Q}$ of quantum tunneling processes is ${ }^{2}$

$$
\Gamma_{Q}-v \exp \left(-U / k_{B} T_{c}\right) .
$$

Therefore, quantum transitions will reveal themselves on the time scale of the experiment if the following condition is satisfied:

$$
k_{B} T<U / 30 \leqslant k_{B} T_{c} .
$$

This means that the barriers involved in the quantum tunneling of magnetization belong to the tale of the distribution of barriers having an energy much lower than the average energy which determines the blocking temperature $T_{B}$.

The theoretical prediction of $T_{c}^{2}$ for particles with uniaxial anisotropy when the external field is applied perpendicular to the axis is

$$
K_{B} T_{C}=\frac{1}{4} \mu_{B} H_{0}\left(1-H / H_{0}\right)^{1 / 2} \text {. }
$$

It corresponds to the temperature range $T_{c} \sim 0.1-5 \mathrm{~K}$ for typical values of the high magnetic anisotropy in very small magnetic particles.

Supposing we have a set of identical noninteracting single domain particles frozen in a nonmagnetic matrix, in presence of a magnetic field. As the field is removed, the total magnetic moment of that system will decay with time according to

$$
M(t)=M(0) e^{-\Gamma t},
$$




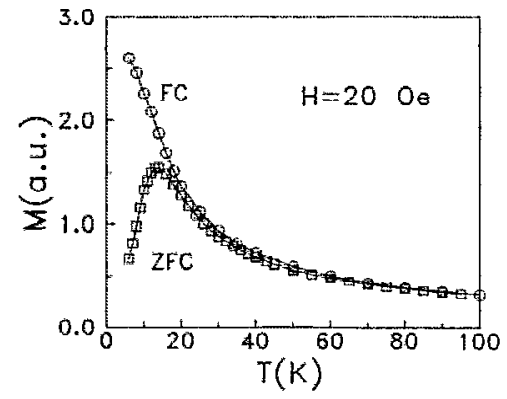

FIG. 1. $M_{\mathrm{FC}}$ and $M_{\mathrm{ZFC}}$ curves for the FeC ferrofluid with an applied field of $H=20 \mathrm{Oe}$.

with $\Gamma$ given by Eq. (2) for thermal processes.

In practice, however, it is very difficult to prepare a system of noninteracting particles, or identical energy barriers. The barrier is proportional to the volume $V$ involved in the tunneling process. Therefore, at $T \rightarrow 0$, the tunneling rate can be presented as

$$
\Gamma_{0}=v \exp \left(-B_{0} r^{3} / r_{0}^{3}\right),
$$

where $v$ is the linear size of the tunneling volume, $r_{0}$ is the size of the volume corresponding to the WKB exponent $B_{0}$. Taking into account that a typical value of $B_{0}$ dictated by the time of the experiment is $B_{0} \approx 30$, the tunneling rate of Eq. (6) will change by the order of magnitude by corresponding a size distribution within only $3 \%$ around $r_{0}{ }^{5}$

In the case of having a broad distribution of energy barriers, the predicted relaxation law ${ }^{6,7}$ for the intensity of magnetization is

$$
M(t)=M_{0}\left[1-S(T) \ln \left(t / t_{0}\right)\right],
$$

where $M_{0}$ is the magnetization at $t_{0}$ and $S(T)$ is the socalled magnetic viscosity. Equation (8) describes the low temperature relaxation due to transition through small barriers, at the tale of the distribution. One should expect this equation to be invalid at large $t$ for $T \sim T_{B}$ (blocking temperature). As we have done before for the escape rate $\Gamma$ of individual events, we define two viscosities: $S_{T}$ (thermal viscosity) and $S_{Q}$ (quantum viscosity). At high temperatures the switching processes are thermally activated and

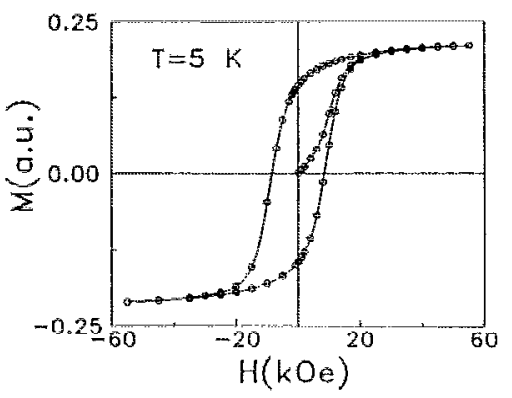

FIG. 2. Hysteresis loop on the $\mathrm{CoFe}_{2} \mathrm{O}_{4}$ ferrofluid sample at $T=5 \mathrm{~K}$.

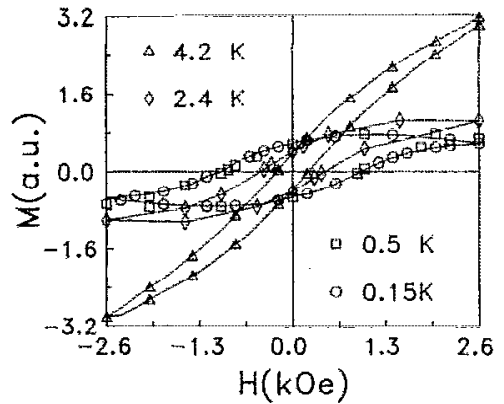

FIG. 3. Hysteresis loops on the FeC ferrofluid sample at temperatures down to $150 \mathrm{mK}$.

$S(T) \sim T$, but below $T_{c}$ the switching process has a quantum nature and consequently $S_{Q}$ is independent of temperature and does not vanish at $T=0 . .^{6,8}$

The constancy of $S_{Q}$ does not depend on the quantum mechanism involved in the relaxation of the magnetization.

The three ferrofluid samples used in this work contain small particles of $\mathrm{FeC}, \mathrm{CoFe}_{2} \mathrm{O}_{4}$, and $\mathrm{Fe}_{3} \mathrm{O}_{4}$, respectively. The electron microscopy characterization of the samples reflects that the particles are basically spherical with a mean particle diameter of $36 \AA(\mathrm{FeC}), 60 \AA\left(\mathrm{Fe}_{3} \mathrm{O}_{4}\right)$ and $50 \AA\left(\mathrm{CoFe}_{2} \mathrm{O}_{4}\right)$. In order to avoid clustering each particle was coated with a nonmagnetic surfactant layer.

The behavior of magnetization versus temperature $T$ in low field was investigated in the zero field cooled (ZFC) and field cooled (FC) states (see Fig. 1). The remarkable feature of the ZFC curve is the existence of a peak at $T=T_{B}$ due to the random orientations of the anisotropy directions below $T_{B}$. The two curves $M_{\mathrm{FC}}$ and $M_{\mathrm{ZFC}}$ are the same for temperatures $T>T_{B}$ with magnetization following a Curie-Weiss law with a very small Curie temperature indicative of the weak magnetic coupling between the particles.

The $M(H)$ curves at $T>T_{B}$ are well fitted to Langevin functions and scale in $H / T$, confirming the superparamagnetic behavior of the ferrofluid particles at $T>T_{B}$. For $T<T_{B}$ these systems present magnetic hysteresis phenomena and one can get information of the energy barrier distribution in the single domain particles by looking at the variation of both coercive field and remanent magnetiza-

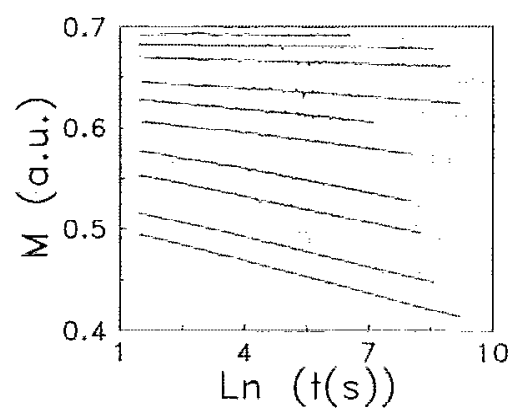

FIG. 4. Time dependence [using criterium $(i)$ ] for the thermoremanence magnetization at different temperatures for $\mathrm{CoFe}_{2} \mathrm{O}_{4}$. The sample was cooled in a field $H_{1}=100 \mathrm{Oe}$ and the relaxation was collected with $H_{2}=0$. 


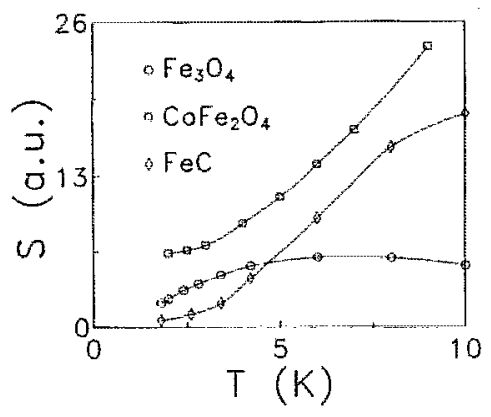

FIG. 5. Temperature dependence of the magnetic viscosity $S$ for the three ferrofluid samples (a) $\mathrm{FeC}$, (b) $\mathrm{Fe}_{3} \mathrm{O}_{4}$, (c) $\mathrm{CoFe}_{2} \mathrm{O}_{4}$.

tion with temperature. In Fig. 2 we show the $M(H)$ data for the $\mathrm{CoFe}_{2} \mathrm{O}_{4}$ sample at $T=5 \mathrm{~K}$. The hysteresis loops on our $\mathrm{FeC}$ sample at temperatures down to $150 \mathrm{mK}$ are shown in Fig. 3. As can be observed in Fig. 3, the coercive field $H_{c}$, is independent of temperature below $0.5 \mathrm{~K}$ suggesting the existence of irreversible changes without thermal activation.

We have studied the time dependence of the magnetization using two different criteria: ${ }^{9-12}$

(i) the sample is cooled at a constant field $H_{1}$ from high temperature to a well-defined final temperature, then the field is switched to a new value $H_{2}$ and relaxation measurements as a function of time are collected. ${ }^{10}$

(ii) After saturation of the magnetization of the sample, the field is reversed and stabilized at a given value $H$ close to the coercive field $H_{c}$, and relaxation measurements as a function of time are collected until the magnetization goes to zero. ${ }^{11}$

Let us list the major features of the relaxation processes.

(1) Relaxation follows the $\log (t)$ law (see Fig. 4) in agreement with the fact of having a broad distribution of energy barriers which are related to the orientation of the particles with respect to the applied field and the effective anisotropy axes.

(2) $S(T)$ presents a maximum at $T \approx T_{B}$ and above $T_{B}$ the time interval for which $M \sim \ln (t)$ is reduced. All of this agrees well with the interpretation of $T_{B}$ as the temperature separating the superparamagnetic and blocked regime (see Fig. 5).

(3) $S(T) \propto T$ at temperatures $T<T_{B}$ which suggests that we are detecting the relaxation corresponding to low barriers. This may also indicate weak correlations between the single domain particles.

(4) $S(T)$ tends to be independent of $T$ for the $\mathrm{CoFe}_{2} \mathrm{O}_{4}$ sample below $3 \mathrm{~K}$ suggesting the occurrence of the quantum tunneling of magnetization at $T<3 \mathrm{~K}$.

The theory (2) suggests that $T_{c}$ depends strongly on

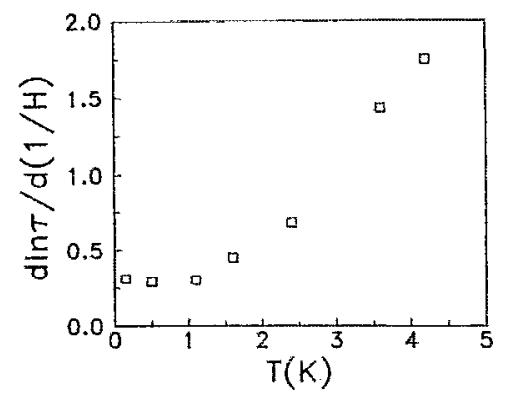

FIG. 6. Dependence of $d \ln \tau / d(1 / H)$ on temperature, with $\tau$ the relaxation time of the switching process and $H$ the applied magnetic field.

the anisotropy field, $H_{k}$ of the particle $\left(k_{B} T_{c} \sim \mu_{B} H_{k}\right)$. Then we can estimate $T_{c}$ for our three samples by substituting the experimental values of $H_{k}$. We have that $T_{c}\left(\mathrm{Fe}_{3} \mathrm{O}_{4}\right) \approx 0.5 \mathrm{~K}, T_{c}(\mathrm{Fe}) \approx 1 \mathrm{~K}$, and $T_{c}\left(\mathrm{CoFe}_{2} \mathrm{O}_{4}\right)=2.5$ $\mathrm{K}$.

Therefore, the plateau in the $S(T)$ values for the $\mathrm{CoFe}_{2} \mathrm{O}_{4}$ sample below $3 \mathrm{~K}$ agrees well with the occurrence of nonthermal, quantum in nature, switching processes of the magnetization at $T<3 \mathrm{~K}$.

(5) The variation with temperature of the mean relaxation time, $\tau$, separating the two magnetic states of $M=M_{R}$ (remanent magnetization) and $M=0$ for the $\mathrm{FeC}$ sample, also presents a plateau below $1 \mathrm{~K}$ (see Fig. 6 and Ref. 9) in agreement with the theoretical prediction of $T_{c}$ for this sample.

As a conclusion, the magnetic relaxation measured at low temperature in three different ferrofluid particles strongly suggest the occurrence of quantum tunneling of the magnetization below a temperature $T_{c}$ which scales with the anisotropy field of these particles.

${ }^{1}$ A. J. Leggett, Prog. Theor. Phys. (Suppl.) 69, 80 (1980); Contemp. Phys. 25, 583 (1984).

${ }^{2}$ E. M. Chudnovsky and L. Gunther, Phys. Rev. Lett. 60, 661 (1988); Phys. Rev. B 37, 9455 (1988).

${ }^{3}$ L. Néel, Ann. Geophys. 5, 99 (1949).

${ }^{4}$ C. P. Bean and J. D. Livingston, J. Appl. Phys. 30 (Suppl.), 1205 (1959).

${ }^{5}$ D. D. Awschalom, J. F. Smyth, G. Grinstein, D. P. Divincenzo, and D. Loss, Phys. Rev. Lett. 68, 3092 (1992)

${ }^{6}$ R. Street and J. C. Wooley, Proc. Phys. Soc. A62, 562 (1949).

${ }^{7} \mathrm{M}$. Uehara and B. Barbara, J. Physique 47, 235 (1986), and references herein.

${ }^{8}$ The theoretical prediction for $S(T)$ is that it must have a universal behavior similar to $\Gamma(T)$ with a plateau below $T_{c}$.

${ }^{9}$ L1. Balcells, J. L. Tholence, S. Linderoth, B. Barbara, and J. Tejada, Z. Phys. B: Condensed Matter (accepted); X. Zhang, Ll. Balcells, J. M. Ruiz, O. Iglesias, and J. Tejada. Phys. Lett. A163, 130 (1992).

${ }^{10}$ X. X. Zhang, LI. Balcelts, J. M. Ruiz, J. L. Tholence, B. Barbara, and J. Tejada, J. Phys.: Condens. Matter 4, L163 (1992).

"For more details, see the invited paper "Nonthermal Viscosity in Magnets: Quantum Tunneling of the Magnetization" by J. Tejada et al.

${ }^{12}$ For more details see the invited paper of B. Barbara et al. in the Symposium on Macroscopic Quantum Tunneling. 